\title{
A Novel Kinematic Parameters Identification Method for Articulated Arm Coordinate Measuring Machines Using Repeatability and Scaling Factor
}

\author{
Lin Cheng $\left(\mathbb{D}\right.$, Wen Wang $\mathbb{D}^{\mathbb{D}}$, Yixin Weng, Guang Shi, He Yang, and Keqing Lu \\ School of Mechanical Engineering, Hangzhou Dianzi University, Hangzhou 310018, China \\ Correspondence should be addressed to Wen Wang; wangwn@hdu.edu.cn
}

Received 18 January 2018; Accepted 27 June 2018; Published 15 July 2018

Academic Editor: Salvatore Strano

Copyright (C) 2018 Lin Cheng et al. This is an open access article distributed under the Creative Commons Attribution License, which permits unrestricted use, distribution, and reproduction in any medium, provided the original work is properly cited.

Kinematic parameters identification and compensation are effective ways to improve the accuracy of articulated arm coordinate measuring machines (AACMMs) and robotic arms without increasing the cost of hardware. Generally, kinematic parameters identification methods based on standard references are relatively high in accuracy but time-consuming and not suitable for industrial sites, while kinematic parameters identification methods based on repeatability are flexible and easy to implement but lack reliability in accuracy. A novel kinematic parameters identification method for AACMMs using repeatability and scaling factor is proposed in this paper, which combines the advantages of methods based on both standard references and repeatability. Through theoretical analysis and numerical simulations, we found that the commonly used single-point-repeatability-based identification method has problems in identifying the length parameters, which is due to that high repeatability cannot guarantee the accuracy of the kinematic parameters and the measurement accuracy of the AACMM. Further analysis showed that the error of the length parameters is determined by a scaling factor which can be used to remove the error of length parameters. Therefore, a two-step novel kinematic parameters identification method for the AACMMs using repeatability and scaling factor was proposed to get accurate parameters with convenient operation. Experimental studies showed the effectiveness of the proposed identification method, which indicated that $93 \%$ more error in spatial length can be decreased comparing to the traditional method of repeatability-based identification.

\section{Introduction}

The Articulated Arm Coordinate Measuring Machine (AACMM) is a portable coordinate measurement instrument, which transforms the measurement of geometry into the measurement of coordinates $[1,2]$. Due to its flexibility and portability, there is a growing demand for applications at industrial sites [3]. However, as a multi-joint serial structure like robotic arm [4,5] AACMMs have lower accuracy comparing with the normal coordinate measuring machines. Limited accuracy of AACMMs restricts the applications and development $[6,7]$.

Some studies have shown that $95 \%$ errors of the AACMM are due to the errors of the kinematic parameters [8-10]. As the systematic errors, the errors of kinematic parameters can be identified by the identification calculation to improve the accuracy of the AACMM.
According to the kinematic chain characteristics of the AACMM, we can obtain multiple sets of kinematic equations under simple physical constraints without using the external high-precision measurement instrument. The kinematic parameters of the AACMM can be identified by optimization algorithms, and the identified parameters will be input to the controller to compensate the errors. According to different evaluation references of the identification methods, the parameters identification methods for AACMMs can be classified into two types. One is based on repeatability, whose objective functions are evaluated by repeatability error with training data acquired from physical constraints. The other is based on standard references, whose objective functions are evaluated by residual error with training data acquired from standard gauges.

Single-point-repeatability-based identification method is one of the most representative methods of repeatability-based 
identification methods. Wang et al. [11] and Gao et al. [12] used a cone hole, which can restrict the center of the probe in the same position, to collect multiple groups of joint angles by changing the poses AACMMs. Under the constraint of single-point cone hole, the coordinates calculated by different group of joint angles will be the same if the kinematic parameters have no errors. However, this is not the fact for all the kinematic parameters which have errors so far. Generally, the differences of the coordinates calculated are used as the objective function of the optimization algorithm to search the actual kinematic parameters. Single-point-repeatabilitybased identification method only uses a simple calibration tool providing a physical constraint of single-point coordinates, which has high data-sampling efficiency and is suitable for parameters identification at industrial sites. However, this kind of identification method has a limited accuracy because the standard geometric values are not introduced in the identification.

Distance-reference-based identification method is a commonly used standard-reference-based identification method. Santolaria et al. estimated the parameters' error of the AACMM by a spherical gauge with 14 standard balls and a self-centering probe $[13,14]$. Cheng et al. [15] took the homemade standard cone hole rod as the distance reference which was calibrated by high-precision measuring instrument. Then the residual error between the experimental distance and the standard distance, measured from the cone hole rod, was applied as the objective function along with the improved least square method used as the optimization algorithm. And the kinematic parameters of the AACMM were identified. As a standard-reference-based identification method, distancereference-based identification method introduces a standard spatial geometric value; thus the kinematic parameters of the AACMM can be identified definitely theoretically. However, distance-reference-based identification method is more complicated than the single-point cone hole identification method during the sampling process, and it is hard to guarantee the stabilization of calibration tool, which will introduce much more the sampling error. Meanwhile the standard distance of the cone hole rod needs to be measured before identification, which increases the manufacturing costs of the calibration tool and the calibration costs.

Comparing the two kinds of identification methods presented above, standard-reference-based identification method can achieve a higher accuracy for AACMMs, but the identification procedure is complex and not suitable for industrial site. Repeatability-based identification method is more convenient and more efficient, but the identification accuracy is lower.

Based on the working principle of the repeatabilitybased identification method, we studied its working mechanism and found that the commonly used single-pointrepeatability-based identification method has defects in identifying the length parameters, that is, high repeatability cannot guarantee the accuracy of the kinematic parameters and the measurement accuracy of the AACMM. Further analysis showed that the error of the length parameters is determined by a scaling factor which can be used to remove the error of length parameters. To improve the accuracy of AACMMs, a novel two-step identification method was proposed in this paper. The first step is to identify the angular parameters and initial length parameters based on singlepoint-repeatability-based. The second step is to achieve the scaling factor for modifying the length parameters based on standard references. Numerical simulations and practical experiments were carried on to verify the method proposed in this paper.

The paper is organized as follows. Section 2 presents the analysis of repeatability-based identification method and the two-step identification method; the numerical simulations of the scaling factor law and the parameters identification process are introduced in Section 3; and experimental results are given in Section 4. Section 5 provides conclusions.

\section{Scaling Factor of Repeatability-Based Identification}

2.1. Scaling Factor. As shown in Figure 1, theoretically, when the probe of the AACMM is poised in the same singlepoint cone hole, a group of coordinates of the probe can be obtained with a serial of poses. And the groups of coordinates are all the same as the probe never moves. When all the length parameters are scaled in a determined factor, another group of coordinates of the probe with the same serial of poses can be obtained. The second group of coordinates are all the same, too. And they are proportional to the first group of coordinates. Moreover, the proportional factor is equal to the scaling factor. This scaling factor law can be described as when all angle parameters of the AACMM remain unchanged, if all length parameters are scaled by a factor $k$, the coordinates of the probe will be scaled by the same factor $k$. The scaling factor law can be proved as follows.

The kinematic model of AACMM is established according to $\mathrm{D}-\mathrm{H}$ method [16]. The coordinates of the probe are obtained by homogeneous matrix transformations which consist of six joints' kinematic parameters (each joint contains joint angle $\theta_{i}$, linkage length $d_{i}$, joint length $a_{i}$, and torsion angle $\alpha_{i}$ ) and the probe offset parameter $l$, as follows:

$$
\begin{aligned}
& {\left[\begin{array}{l}
x \\
y \\
z \\
1
\end{array}\right]=\prod_{i=1}^{6} T_{i}\left[\begin{array}{l}
0 \\
0 \\
l \\
1
\end{array}\right]} \\
& =\prod_{i=1}^{6}\left[\begin{array}{ccc|c}
\cos \theta_{i} & -\sin \theta_{i} \cos \alpha_{i} & \sin \theta_{i} \sin \alpha_{i} & a_{i} \cos \theta_{i} \\
\sin \theta_{i} & \cos \theta_{i} \cos \alpha_{i} & -\cos \theta_{i} \sin \alpha_{i} & a_{i} \sin \theta_{i} \\
0 & \sin \alpha_{i} & \cos \alpha_{i} & d_{i} \\
\hline 0 & 0 & 0 & 1
\end{array}\right]
\end{aligned}
$$

$$
\cdot\left[\begin{array}{l}
0 \\
0 \\
l \\
\hline 1
\end{array}\right]
$$

The transformation matrix $T_{i}$ for adjacent joints can be divided into $2 \times 2$ matrices and all the length parameters are in 


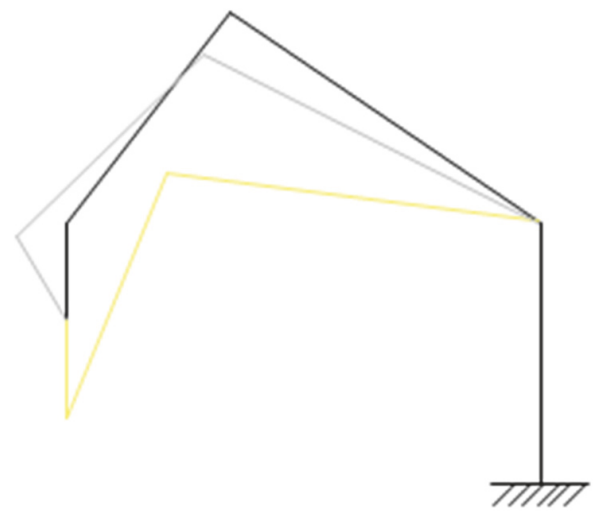

(a) Before scaling

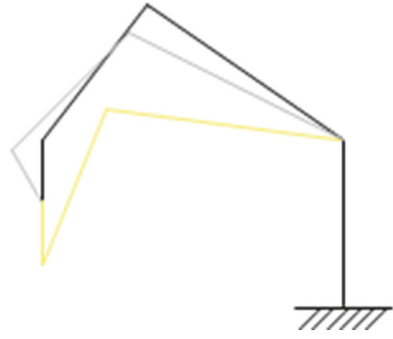

(b) After scaling

FIGURE 1: Scaling with the same factor.

the divided matrix in the upper right corner. The probe offset matrix can be divided into upper and lower matrices, and the length parameter is in the upper matrix, if we set $T_{\mathrm{i}}=\left[\begin{array}{cc}A_{i} B_{i} \\ 0 & 1\end{array}\right]$, $\left[\begin{array}{l}0 \\ 0 \\ l \\ 1\end{array}\right]=\left[\begin{array}{l}C \\ 1\end{array}\right],\left[\begin{array}{l}x \\ y \\ z \\ 1\end{array}\right]=\left[\begin{array}{l}X \\ 1\end{array}\right]$, then $\left[\begin{array}{l}X \\ 1\end{array}\right]=\prod_{i=1}^{6}\left[\begin{array}{cc}A_{i} & B_{i} \\ 0 & 1\end{array}\right] \cdot\left[\begin{array}{l}C \\ 1\end{array}\right]$.

If the length parameters are scaled in the same factor of $k$, we will obtain the following equation:

$$
\begin{aligned}
\mathrm{T}_{\mathrm{i}} \cdot \mathrm{T}_{\mathrm{i}+1} & =\left[\begin{array}{cc}
A_{i} & \mathrm{k} B_{i} \\
0 & 1
\end{array}\right] \cdot\left[\begin{array}{cc}
A_{i+1} & k B_{i+1} \\
0 & 1
\end{array}\right] \\
& =\left[\begin{array}{cc}
A_{i} A_{i+1} & \mathrm{k}\left(B_{i}+A_{i} B_{i+1}\right) \\
0 & 1
\end{array}\right]
\end{aligned}
$$

In the process of transformation, $k$ always exists in the upper right corner of $T_{i}$ and $T_{i+1}$, and it also exists in the upper right corner of the product of $\mathrm{T}_{\mathrm{i}} \cdot \mathrm{T}_{\mathrm{i}+1}$. To multiply the result by the probe offset matrix scaled with same factor, we can get:

$$
\mathrm{T}_{\mathrm{i}} \cdot \mathrm{T}_{\mathrm{i}+1} \cdot\left[\begin{array}{c}
k C \\
1
\end{array}\right]=\left[\begin{array}{c}
k\left(A_{i} A_{i+1} C+B_{i}+A_{i} B_{i+1}\right) \\
1
\end{array}\right]
$$

The scaling factor still exists in the upper partitioned matrix which is linear. It can be deduced that the scaled coordinates of the probe are

$$
\left[\begin{array}{c}
x^{\prime} \\
y^{\prime} \\
z^{\prime} \\
1
\end{array}\right]=\prod_{i=1}^{6}\left[\begin{array}{cc}
A_{i} & k B_{i} \\
0 & 1
\end{array}\right] \cdot\left[\begin{array}{c}
k C \\
1
\end{array}\right]=\left[\begin{array}{c}
k X \\
1
\end{array}\right]=\left[\begin{array}{c}
k x \\
k y \\
k z \\
1
\end{array}\right]
$$

According to (4) we can get the conclusion: when all angle parameters of the AACMM remain unchanged, if all length parameters are scaled by a factor $k$, the coordinates of the probe will be scaled by the same factor $k$. In other words, if the size of the AACMM is scaled $k$, the coordinates of the probe will be scaled $k$ times accordingly.
From the scaling factor law it can be deduced that the distance calculated from the scaled coordinates are also scaled, as shown in (6). Hence, if the length parameters of the AACMM are scaled, there will be a systematic error in measuring the distance.

$$
\begin{aligned}
L & =\sqrt{\left(x_{2}-x_{1}\right)^{2}+\left(y_{2}-y_{1}\right)^{2}+\left(z_{2}-z_{1}\right)^{2}} \\
L^{\prime} & =\sqrt{\left(k x_{2}-k x_{1}\right)^{2}+\left(k y_{2}-k y_{1}\right)^{2}+\left(k z_{2}-k z_{1}\right)^{2}} \\
& =k L
\end{aligned}
$$

2.2. First Step of Identification Method Using Repeatability. The kinematic parameters that determine the coordinates of the probe of the AACMM consist of the length parameters $D$ including linkage length $d_{i}$, joint length $a_{i}$, the probe offset $l$, and the angle parameters $R$ including torsion angle $\alpha_{i}$ and joint angle $\theta_{i}$. The joint angle $\theta_{i}$ is composed of the sensor data $\theta_{s i}$ (experimentally obtained with high accuracy encoders) and the joint zero angle deviation $\theta_{0 i}$. Here we take coordinate $x$, for example,

$$
x_{j}=f_{x}\left(\theta_{j, s i}, D, R\right)
$$

where $j$ is the order of repeated measurements of the same point, $j=1,2, \ldots, n$.

If the length parameters $D$ are equal to the actual length parameters $D_{0}$ and the angle parameters $R$ are equal to the actual angle parameters $R_{0}$, it can be deduced that the coordinate $x_{j}$ calculated by any $j$ th measurement is equal to the real coordinate $x_{0}$. Hence, all the coordinates $x_{j}$ calculated are equal. The standard deviation $\sigma$ that evaluates repeatability is zero:

$$
\left\{\begin{array}{l}
D=D_{0} \\
R=R_{0}
\end{array} \longrightarrow x_{j}=x_{0} \longrightarrow x_{j}=x_{j+1} \longrightarrow \sigma=0\right.
$$

According to the law of scaling factor of length parameters, there is another situation in which standard deviation 
$\sigma$ is also zero. When the length parameter $D$ is equal to $k D_{0}$ (i.e., $k$ times the actual length parameters $D_{0}$ ), the angle parameters $R$ are equal to the actual angle parameters $R_{0}$. Furthermore, it can be deduced that the coordinate $x_{j}$ calculated by any $j$ th measurement is equal to $k x_{0}$ ( $k$ times the real coordinate). Also the values of all the calculated coordinates $x_{j}$ are still equal. So the standard deviation $\sigma$ is still zero, i.e.,

$$
\left\{\begin{array}{l}
D=k D_{0} \\
R=R_{0}
\end{array} \longrightarrow x_{j}=k x_{0} \longrightarrow x_{j}=x_{j+1} \longrightarrow \sigma=0\right.
$$

It can be inferred that $\sigma=0$ is a necessary but insufficient condition for the identifiability of the kinematic parameters. And the objective function here with the standard deviation can be extended to any other function that evaluates repeatability error.

$$
\left\{\begin{array}{l}
D=D_{0} \\
R=R_{0}
\end{array} \longrightarrow \sigma=0\right.
$$

Therefore, when all angle parameters are unchanged and all length parameters are scaled by $k$ times, the repeatability error is zero. So the repeatability-based identification method cannot accurately identify the kinematic parameters of each joint.

2.3. Second Step of Identification Method Using Scaling Factor Law. According to (4) and (6), if the length parameters of AACMM are scaled with the same factor, the measuring results will contain systematic error. This paper presents a novel kinematic parameters identification method for the AACMMs using repeatability and the law of scaling factor. The novel method includes the repeatability-based identification, and the absolute measuring to get the scaling factor of systematic errors with the distance reference. Firstly, the accurate angle parameters and preliminary length parameters are identified with the repeatability-based identification method. Then, after compensating with the identified parameters, the AACMM are used to measure a gauge to get the scaling factor $k=L^{\prime} / L$, where $L$ is the nominal length of the gauge, and $L^{\prime}$ is the measured length of the gauge. Finally, the accurate length parameters will be obtained by $D^{\prime}=D / k$.

\section{Numerical Simulation}

To verify the method proposed in Section 2, numerical simulations were conducted.

We consider the theoretical kinematic parameters of the

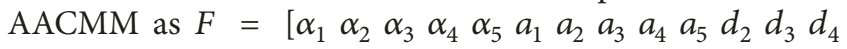
$\left.d_{5} d_{6} \theta_{02} \theta_{03} \theta_{04} \theta_{05} \theta_{06} l\right]=\left[\begin{array}{llll}-1.5708 & -1.5708 & -1.5708\end{array}\right.$ $\begin{array}{lllllllllllllll}-1.5708 & -1.5708 & 0 & 62 & 0 & 62 & 0 & 0 & 750 & 0 & 500 & 11.5 & 0 & 0 & 0\end{array}$ $\left.\begin{array}{lll}0 & 0 & 102\end{array}\right]$.

According to the kinematic model and the theoretical kinematic parameters of the AACMM, 70 groups of joint angles $\theta_{s i}$ (i.e., different poses) are obtained from the assumed coordinates of single-point position.

According to definitions in Certification Standard JJF 1408-2013 [17] and ASME B.89.4.22 standard (hereinafter referred to as the certification standard), the errors in $x, y$, and $z$ directions are

$$
\begin{aligned}
& \delta_{x}=\frac{\max \left(x_{j}\right)-\min \left(x_{j}\right)}{2} \\
& \delta_{y}=\frac{\max \left(y_{j}\right)-\min \left(y_{j}\right)}{2} \\
& \delta_{z}=\frac{\max \left(z_{j}\right)-\min \left(z_{j}\right)}{2}
\end{aligned}
$$

The single-point repeatability accuracy is designed as

$$
\text { Deltam }=\max \left(\delta_{x}, \delta_{y}, \delta_{z}\right)
$$

The single-point repeatability accuracy of the AACMM calculated by (11) and (12) with the 70 groups of simulation data is $3 \times 10^{-6} \mathrm{~mm}$.

3.1. The Effects of Scaling Factor on Repeatability Accuracy. We can scale the length parameters with a scaling factor of $1 / 2$, i.e., $F=\left[\begin{array}{lllll}-1.5708 & -1.5708 & -1.5708 & -1.5708 & -1.5708\end{array}\right.$ $\begin{array}{llllllllllllllll}0 & 31 & 0 & 31 & 0 & 0 & 375 & 0 & 250 & 5.75 & 0 & 0 & 0 & 0 & 0 & 51] .\end{array}$

The single-point repeatability accuracy of the AACMM calculated by (11) and (12) with the 70 groups of simulation data is $2 \times 10^{-6} \mathrm{~mm}$. The deviation between the repeatability accuracies before and after scaling length parameters can be neglected with regard to the normal accuracy $\left(5 \times 10^{-2} \mathrm{~mm}\right)$ of AACMMs. This numerical simulation suggests that the scaling factor of the length parameters has little effect on the single-point repeatability accuracy of the AACMM.

3.2. Simulation of Single-Point-Repeatability-Based Identification. The coordinates $\left(x_{j}, y_{j}, z_{j}\right)$ of all measuring points are calculated according to homogeneous matrix transformation formula (1), where $j=1,2, \ldots, N$. The average coordinates of the measuring points in $x, y$, and $z$ directions are as follows:

$$
\begin{aligned}
& \bar{x}=\frac{1}{N} \sum_{\mathrm{j}=1}^{N} x_{j}, \\
& \bar{y}=\frac{1}{N} \sum_{\mathrm{j}=1}^{N} y_{j}, \\
& \bar{z}=\frac{1}{N} \sum_{\mathrm{j}=1}^{N} z_{j}
\end{aligned}
$$

The error distance of each measuring point from the average coordinate point can be derived:

$$
E_{j}=\sqrt{\left(x_{j}-\bar{x}\right)^{2}+\left(y_{j}-\bar{y}\right)^{2}+\left(z_{j}-\bar{z}\right)^{2}}
$$

The mean error distance of all measuring points can be calculated, which can represent the repeatability accuracy of the AACMM:

$$
D E=\frac{1}{N} \sum_{\mathrm{j}=1}^{N} E_{j}
$$




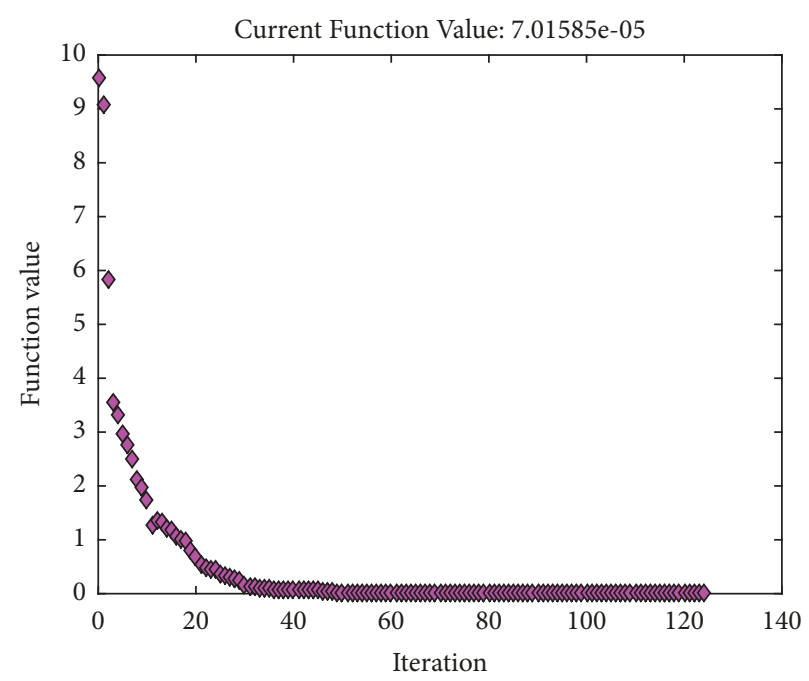

FIgUre 2: Simulation identification results.

When the kinematic parameters are unknown, we carry out the identification with D-H model and the joint angles. Here we use 70 groups of joint angles as the input and use the mean error distance $D E$ as the objective function according to (15) with the optimization algorithm of the interior point.

(1) The lower and upper boundaries of the variables (kinematic parameters) to be searched are set to be: $l b=\left[\begin{array}{llllllll}-1.5883 & -1.5883 & -1.5883 & -1.5883 & -1.5883 & -10 & 52 & -10\end{array}\right.$ $\begin{array}{llllllllll}52 & -10 & -10 & 740 & -10 & 490 & 1.5 & -0.0175 & -0.0175 & -0.0175\end{array}$ $-0.0175-0.017592]$ and $u b=\left[\begin{array}{llll}-1.5533 & -1.5533 & -1.5533\end{array}\right.$ $\begin{array}{llllllllllll}-1.5533 & -1.5533 & 10 & 72 & 10 & 7210 & 10 & 760 & 10 & 510 & 21.5 & 0.0175\end{array}$ $\left.\begin{array}{llll}0.0175 & 0.0175 & 0.01750 .0175 & 112\end{array}\right]$.

The kinematic parameters obtained through identification are as follows:

$$
\begin{aligned}
F= & {\left[\begin{array}{llllll}
-1.570801 & -1.570798 & -1.570804 & -1.570798 \\
& -1.570797 & 0.000103 & 61.966195 & 0.001734 & 61.966583 \\
& 0.000112 & -0.000039 & 749.597077 & 0.000363
\end{array}\right.}
\end{aligned}
$$$$
499.730124 \quad 11.493096 \quad-0.000001 \quad 0.000000
$$

$\left.\begin{array}{llll}0.000004 & 0.000002 & 0.000000 & 101.945075\end{array}\right]$.

The results of the identification are shown in Figure 2. The mean error distance $D E$ is $7 \times 10^{-5} \mathrm{~mm}$, and single-point repeatability accuracy Deltam is $3.7 \times 10^{-4} \mathrm{~mm}$ according to the certification standard.

(2) Keeping the searching range of the angle parameters unchanged, the searching center of the length parameters deviate is set to $+5 \mathrm{~mm}$ :

$l b=\left[\begin{array}{lllllll}-1.5883 & -1.5883 & -1.5883 & -1.5883 & -1.5883 & -5 & 57\end{array}\right.$ $\begin{array}{lllllllllll}-5 & 57 & -5 & -5 & 745 & -5 & 495 & 6.5 & -0.0175 & -0.0175 & -0.0175\end{array}$ $-0.0175-0.017597]$ and $u b=\left[\begin{array}{llll}-1.5533 & -1.5533 & -1.5533\end{array}\right.$ $\begin{array}{lllllllllllll}-1.5533 & -1.5533 & 15 & 77 & 15 & 77 & 15 & 15 & 765 & 15 & 515 & 26.5 & 0.0175\end{array}$ $\left.\begin{array}{lllll}0.0175 & 0.0175 & 0.0175 & 0.0175 & 117\end{array}\right]$.
The identified kinematic parameters are as follows:

$$
\begin{aligned}
& F=\left[\begin{array}{lll}
-1.570799 & -1.570802 & -1.570800
\end{array}\right. \\
& -1.570797-1.570801 \\
& \begin{array}{llll}
-0.001005 & 62.547732 & -0.001035 & 62.547997
\end{array} \\
& \begin{array}{lll}
-0.000070 & -0.000025 & 756.618307
\end{array} \\
& -0.000419504 .41256611 .601591 \\
& \begin{array}{lll}
-0.000001 & -0.000002 & -0.000001
\end{array} \\
& \begin{array}{lll}
-0.000001 & 0.000000 & 102.900101] .
\end{array}
\end{aligned}
$$

As identification results shown in Figure 3, the mean error distance $D E$ is $8 \times 10^{-5} \mathrm{~mm}$, and single-point repeatability accuracy Deltam can be calculated as $1.5 \times 10^{-4} \mathrm{~mm}$ according to the certification standard.

Two simulations of single-point-repeatability-based identification are compared in Table 1.

Although the searching boundaries of the two groups of variables are different, the single-point repeatability accuracies of the two identification results are both in the range of $10^{-4} \mathrm{~mm}$. Hence, the difference between the two accuracies can be neglected. However, by comparing each kinematic parameter of the results of simulation identifications with the theoretical kinematic parameters, we know the maximum error of the length parameters is $0.4 \mathrm{~mm}$ in the first simulation and $6.6 \mathrm{~mm}$ in the second simulation. It should be noted that the errors of the angle parameters may be neglected. Therefore, it suggests that while the objective function of repeatability error (accuracy) converges, the obtained length parameters will have different factor scaling with different settings of searching boundaries.

In addition, after a serial of numerical simulations, it is found that the scaling of the length parameters will decrease when the ranges of parameters are small. But in practical identification experiments, it is impractical to set the 


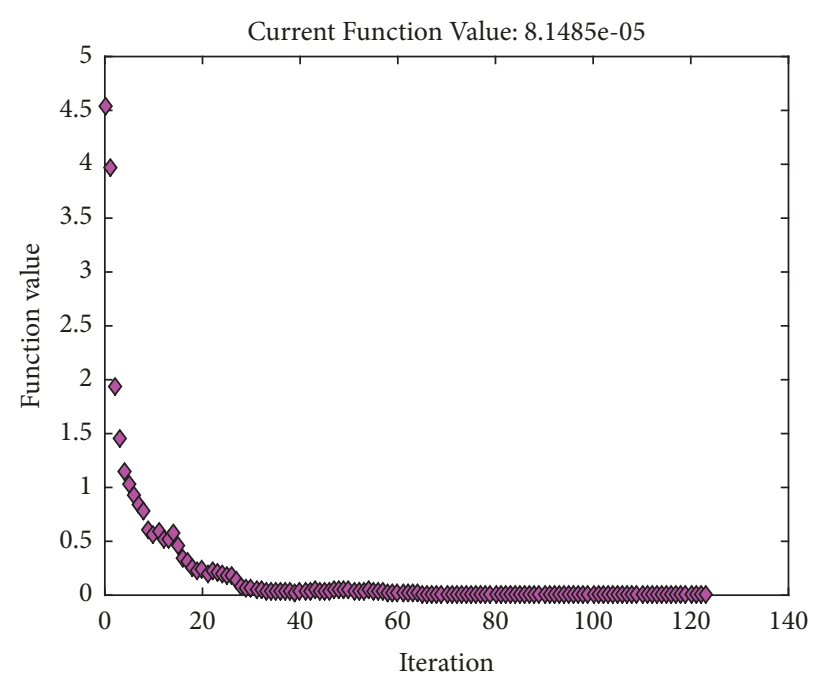

FIGURE 3: Simulation identification results after changing the searching range.

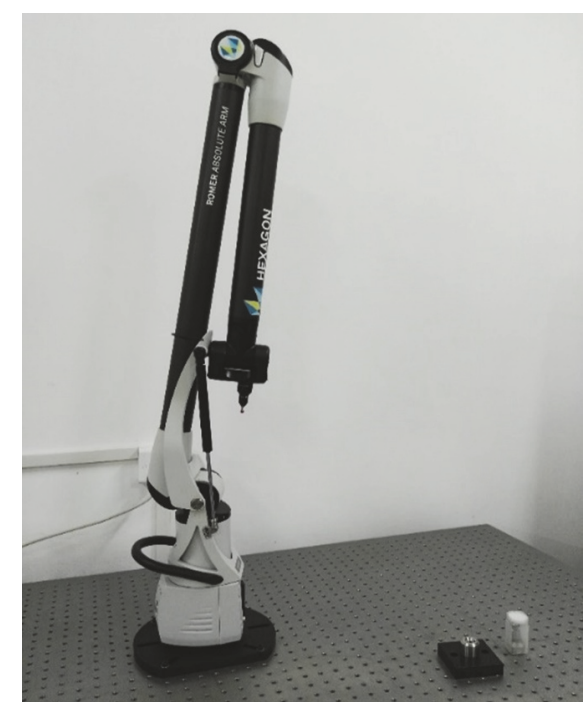

Figure 4: Hexagon RA7125.

ranges of parameters small for the actual parameters that are unknown. Once the small ranges are set incorrectly, the identification algorithms cannot achieve the actual parameters.

The above numerical simulations illustrate that the identification using the repeatability error as the objective function cannot assure the accuracy of the obtained length parameters. In general, the length parameters obtained by repeatabilitybased identification should be scaled with same factor compared with the nominal values.

\section{Experiments}

To verify the novel kinematic parameters identification method and scaling factor law, experiments were conducted. We carried out the repeatability-based identification, following with obtaining scaling factor of systematic error by measuring the distance reference, then compensating the length parameters by using the scaling factor.
4.1. Single-Point-Repeatability-Based Identification Experiment. The AACMM of Hexagon RA7125 was used in the experiment at the environmental temperature of $20^{\circ} \mathrm{C} .70$ groups of joint angles ("Datal") were collected at the same single-point position in different poses with the constraint of the three-ball cone hole, as shown in Figures 4 and 5 .

Like the parameters identification in the simulation, we used 70 groups of joint angles as the input with the mean error distance $D E$ as the objective function according to (15).

The lower searching boundary and the upper searching boundary of the kinematic parameters were set as follows:

$l b=\left[\begin{array}{llllllll}-1.5883 & -1.5883 & 1.5533 & -1.5883 & 1.5533 & -10 & 52 & -10\end{array}\right.$ $\begin{array}{llllllllll}52 & -10 & -10 & 740 & -10 & 490 & 5 & -0.0175 & -0.0175 & -0.0175\end{array}$

$\left.\begin{array}{lll}-0.0175 & -0.0175 & 87\end{array}\right]$ and $u b=\left[\begin{array}{llll}-1.5533 & -1.5533 & 1.5883\end{array}\right.$

$\begin{array}{lllllllllllll}-1.5533 & 1.5883 & 10 & 72 & 10 & 72 & 10 & 10 & 760 & 10 & 510 & 25 & 0.0175\end{array}$

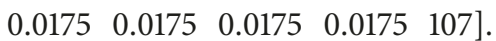


TABLE 1: Comparison of identifications in simulation.

\begin{tabular}{|c|c|c|c|c|c|c|}
\hline $\begin{array}{l}\text { No. of } \\
\text { simulations }\end{array}$ & $\begin{array}{l}\text { Length } \\
\text { searching range } \\
{[\mathrm{mm}]}\end{array}$ & $\begin{array}{c}\text { Angle searching } \\
\text { Range }\left[{ }^{\circ}\right]\end{array}$ & $\begin{array}{c}\mathrm{DE} \\
{[\mathrm{mm}]}\end{array}$ & $\begin{array}{l}\text { Deltam } \\
{[\mathrm{mm}]}\end{array}$ & $\begin{array}{c}\text { Length Max } \\
\text { Error }[\mathrm{mm}]\end{array}$ & $\begin{array}{c}\text { Angle Max } \\
\text { Error }\end{array}$ \\
\hline 1 & {$[-10,+10]$} & {$[-1,+1]$} & $7 \times 10^{-5}$ & $3.7 \times 10^{-4}$ & 0.4 & $4 \times 10^{-6}$ \\
\hline 2 & {$[-5,+15]$} & {$[-1,+1]$} & $8 \times 10^{-5}$ & $1.5 \times 10^{-4}$ & 6.6 & $3 \times 10^{-6}$ \\
\hline
\end{tabular}

TABLE 2: The kinematic parameters identified of the AACMM.

\begin{tabular}{|c|c|c|c|c|}
\hline Joint number $i$ & $\alpha_{i}\left[^{\circ}\right]$ & $a_{i}[\mathrm{~mm}]$ & $\theta_{0 i}\left[{ }^{\circ}\right]$ & $d_{i}[\mathrm{~mm}]$ \\
\hline 1 & -89.9683 & -0.0226 & 0 & 376.5 \\
\hline 2 & -90.0312 & 62.1163 & 0.0040 & -0.0525 \\
\hline 3 & 90.0071 & -0.0061 & 0.0466 & 750.7422 \\
\hline 4 & -90.0338 & 62.1025 & -0.0374 & 0.0597 \\
\hline 5 & 90.0373 & 0.0049 & 0.0127 & 500.5566 \\
\hline 6 & -90 & 0 & -0.0060 & 15.0695 \\
\hline \multicolumn{5}{|c|}{ Probe offset $l=97.8110 \mathrm{~mm}$} \\
\hline
\end{tabular}

TABLE 3: Single-point repeatability accuracy verification by different datasets.

\begin{tabular}{llll}
\hline Datasets (70 groups) & "Datal" & "Data2" & "Data3" \\
\hline $\begin{array}{l}\text { Single-point repeatability } \\
\text { accuracy/mm }\end{array}$ & 0.0187 & 0.0326 & 0.0274 \\
\hline
\end{tabular}

The identified kinematic parameters are as follows:

$$
\begin{aligned}
& F=\left[\begin{array}{lll}
-1.570742 & -1.570856 & 1.570870
\end{array}\right. \\
& \begin{array}{llll}
-1.570865 & 1.571311 & -0.022551 & 61.883904
\end{array} \\
& \begin{array}{lll}
-0.006059 & 61.870128 & 0.004900
\end{array} \\
& \begin{array}{lllll}
-0.052339 & 747.933132 & 0.059462 & 498.683691 & 15.013116
\end{array} \\
& \begin{array}{llll}
-0.000160 & 0.000009 & 0.000104 & 0.000172
\end{array} \\
& -0.000069 \text { 97.445020]. }
\end{aligned}
$$

As shown in Figure 6, after single-point-repeatabilitybased identification, the mean error distance $D E$ is $0.0095 \mathrm{~mm}$, and single-point repeatability accuracy Deltam is $0.0186 \mathrm{~mm}$ according to the certification standard.

4.2. System Error Compensation. A gauge was measured four times with the AACMM after single-point-repeatabilitybased identification, whose nominal length is of $201.513 \mathrm{~mm}$. The measuring results were as follows: $200.761 \mathrm{~mm}$, $200.759 \mathrm{~mm}, 200.755 \mathrm{~mm}$, and $200.759 \mathrm{~mm}$, so the average is $200.759 \mathrm{~mm}$ with the deviation of $0.006 \mathrm{~mm}$ between maximum and minimum, and maximum measurement error is $0.758 \mathrm{~mm}$. According to the analysis in Section 2, the systematic errors are caused by the scaling factor of the length parameters in the repeatability-based identification.

By (6), we know the scaling factor $k=200.759 / 201.513$. Then, the accurate length parameters $D^{\prime}=D / k$ and the final kinematic parameters can be calculated which is shown in Table 2.

4.3. Single-Point Repeatability Accuracy Verification. Another two groups of single-point data were used to verify the single-point repeatability accuracy of the identified kinematic parameters. As shown in Table 3, the average is $0.026 \mathrm{~mm}$.

4.4. Spatial Length Accuracy Verification. According to the certification standard, the spatial length deviation is

$$
\operatorname{Dev}=L_{j}-L_{\text {cal }}
$$

where $L_{j}$ is the result of each length measurement, $L_{c a l}$ is actual length value.

The maximum spatial length deviation is designed as

$$
\text { MaxDev }=\max (|\operatorname{Dev}|)
$$

As shown in Figure 7, we used the AACMM after compensation with the parameters of Table 2 to measure a quartzes plate (the nominal length of $300.0005 \mathrm{~mm}$ ) 30 times, which was placed in different positions and orientations. The results of measurements were shown in Figure 8 . The maximum value is $300.036 \mathrm{~mm}$, the minimum measured value is $299.950 \mathrm{~mm}$, the maximum deviation between the measured value and the standard value is $0.050 \mathrm{~mm}$, the range of the deviations is $0.086 \mathrm{~mm}$, and the two times the rootmean-square of the deviations is $0.061 \mathrm{~mm}$.

According to the certification standard, the single-point repeatability of the AACMM finally calibrated in this research is $0.026 \mathrm{~mm}$ and the spatial length accuracy is $\pm 0.050 \mathrm{~mm}$, which has $93 \%$ improvement in length accuracy compared with traditional method of repeatability-based identification with no length scaling compensation.

\section{Conclusions}

Repeatability-based identification methods are convenient and efficient in acquiring data and identification calculation, 


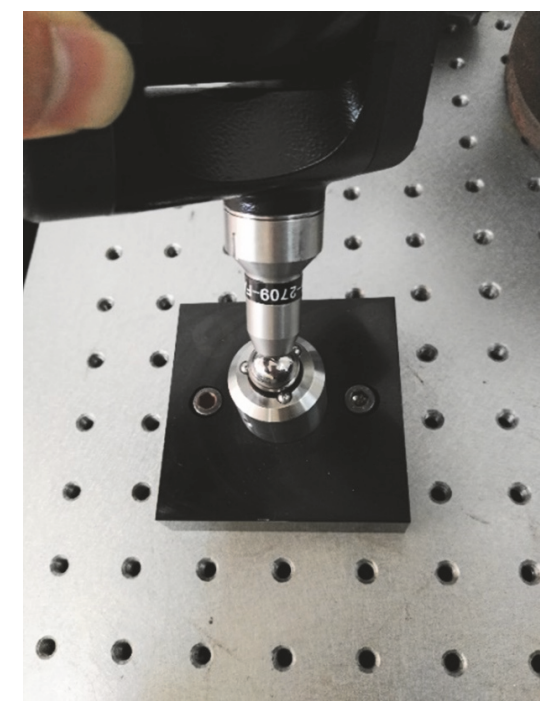

FIGURE 5: Single-point data acquisition.

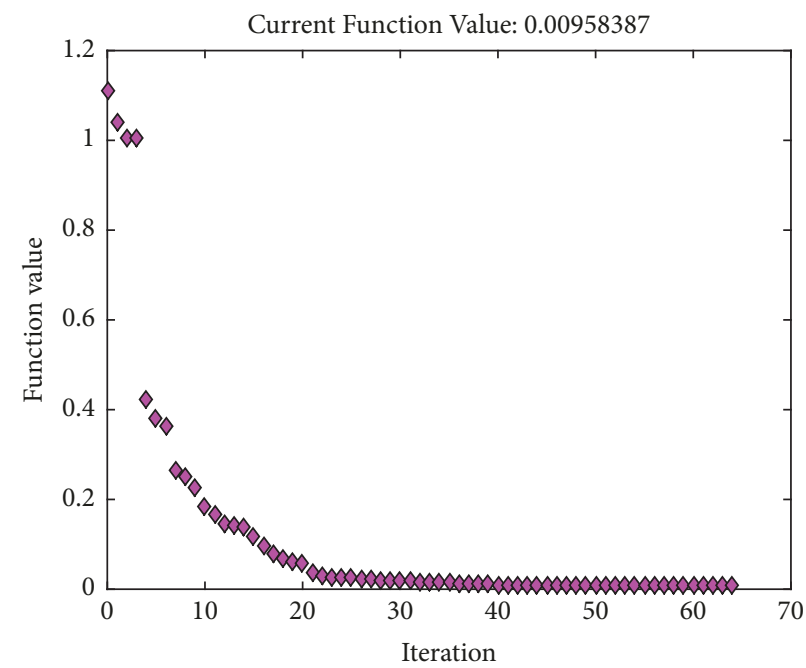

FIGURE 6: The result of single-point-repeatability-based identification.

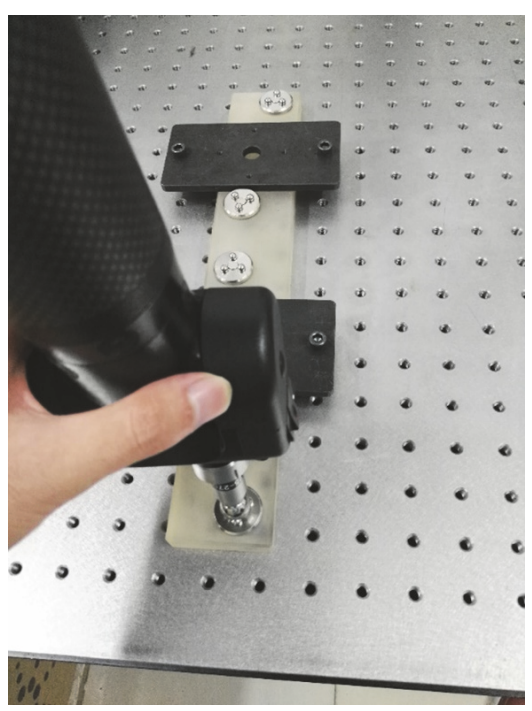

FIGURE 7: Length accuracy verification experiment. 


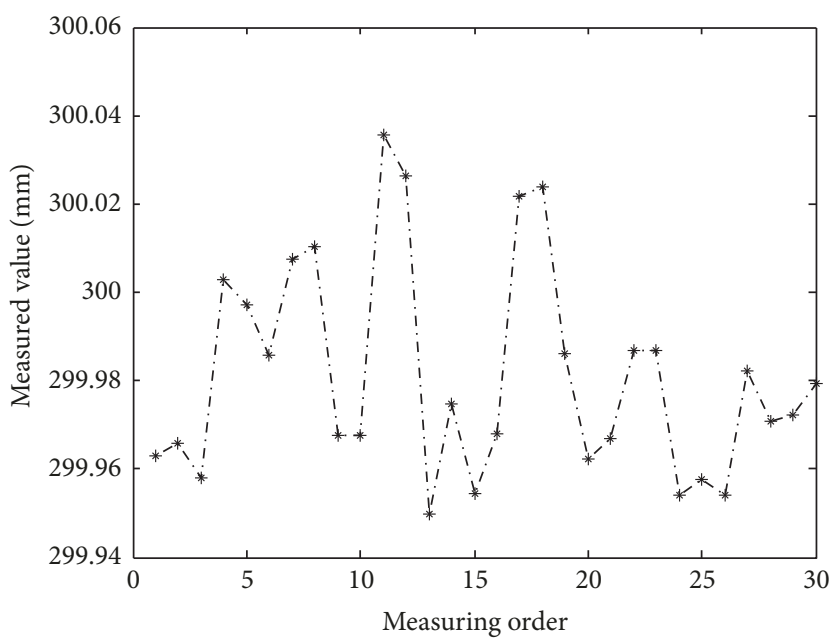

FIgURE 8: Length accuracy verification results.

but they are low in accuracy. This limits their applications in the field of AACMMs greatly. Through theoretical analysis and numerical simulations, we found that the length parameters of AACMMs cannot be identified accurately by repeatability-based identification method for there being a scaling factor law in the repeatability-based identification. And accurate kinematic parameters cannot be identified without introducing any standard reference. Therefore, a twostep novel kinematic parameters identification method for the AACMMs using repeatability and scaling factor is proposed in this paper to get accurate parameters by convenient operation. Experimental studies have verified the feasibility of the proposed identification method, which indicates that $93 \%$ more error in spatial length can be decreased comparing to the traditional method of repeatability-based identification. Therefore, the accuracy of the AACMM is improved greatly.

The scaling factor problem also exists in the kinematic identifications of similar linkage mechanisms, e.g., industrial robots. The novel parameters identification method proposed in this paper also can be applied in the parameters identification for industrial robots to improve the accuracy of them.

\section{Data Availability}

The [text] data used to support the findings of this study have been deposited in the [https://figshare.com/] repository ([https://doi.org/10.6084/m9.figshare.6223811.v1]).

\section{Conflicts of Interest}

The authors declare that they have no conflicts of interest.

\section{Acknowledgments}

This work was supported by the National Natural Science Foundation of China (Grant no. 51465027), Zhejiang Provincial Natural Science Foundation of China (Grant no. LZ16E050001), and the Key Laboratory of Advanced Manufacturing Technology of Zhejiang Province.

\section{References}

[1] A. Piratelli-Filho, F. H. T. Fernandes, and R. V. Arencibia, "Application of virtual spheres plate for AACMMs evaluation," Precision Engineering, vol. 36, no. 2, pp. 349-355, 2012.

[2] Guanbin Gao, Hongwei Zhang, Hongjun San, Xing Wu, and Wen Wang, "Modeling and Error Compensation of Robotic Articulated Arm Coordinate Measuring Machines Using BP Neural Network," Complexity, vol. 2017, pp. 1-8, 2017.

[3] J. Santolaria, A. C. Majarena, D. Samper et al., "Articulated arm coordinate measuring machine calibration by laser tracker multilateration," The Scientific World Journal, vol. 2014, Article ID 681853, pp. 1-11, 2014.

[4] B. Benciolini and A. Vitti, "A new quaternion based kinematic model for the operation and the identification of an articulated arm coordinate measuring machine inspired by the geodetic methodology," Mechanism and Machine Theory, vol. 112, pp. 192-204, 2017.

[5] Guanbin Gao, Huaishan Zhang, Xing Wu, and Yu Guo, "Structural Parameter Identification of Articulated Arm Coordinate Measuring Machines," Mathematical Problems in Engineering, vol. 2016, pp. 1-10, 2016.

[6] R. Acero, A. Brau, J. Santolaria, and M. Pueo, "Evaluation of a metrology platform for an articulated arm coordinate measuring machine verification under the ASME B89.4.22-2004 and VDI 2617_9-2009 standards," Journal of Manufacturing Systems, vol. 42, pp. 57-68, 2017.

[7] K. Ostrowska, A. Gąska, R. Kupiec, J. Sładek, and K. Gromczak, "Verification of Articulated Arm Coordinate Measuring Machines Accuracy Using LaserTracer System as Standard of Length," Mapan - Journal of Metrology Society of India, vol. 31, no. 4, pp. 241-256, 2016.

[8] R. P. Judd and A. B. Knasinski, "Technique to calibrate industrial robots with experimental verification," IEEE Transactions on Robotics and Automation, vol. 6, no. 1, pp. 20-30, 1990.

[9] B. Mooring, "The effect of joint axis misalignment on robot positioning accuracy[C]," in Proceedings of the ASME International Computers in Engineering Conference and Exhibition, pp. 151-155, 1983. 
[10] C.-H. Wu, "Kinematic error model for the design of robot manipulator," in Proceedings of the 1983 American Control Conference, pp. 497-502, 1983.

[11] P. Wang, Y. Fei, P. Shang, and W. Cheng, "Parameters identification of a flexible coordinate measuring machine," Nongye Jixie Xuebao/Transactions of the Chinese Society of Agricultural Machinery, vol. 38, no. 7, pp. 129-132, 2007.

[12] G. Gao, J. Na, X. Wu, and Y. Guo, "A self-calibration method for articulated arm coordinate measuring machines," Transactions of the Canadian Society for Mechanical Engineering, vol. 40, no. 4, pp. 645-655, 2016.

[13] J. Santolaria, J.-J. Aguilar, J.-A. Yagüe, and J. Pastor, "Kinematic parameter estimation technique for calibration and repeatability improvement of articulated arm coordinate measuring machines," Precision Engineering, vol. 32, no. 4, pp. 251-268, 2008.

[14] J. Santolaria, A. Brau, J. Velzquez, and J. J. Aguilar, "A self-centering active probing technique for kinematic parameter identification and verification of articulated arm coordinate measuring machines," Measurement Science and Technology, vol. 21, no. 5, 2010.

[15] W. Cheng, L. Yu, and Y. Fei, "Study on parameter identification algorithms for articulated arm coordinate measuring machine," Journal of University of Science and Technology of China, vol. 41, no. 1, pp. 45-49, 2011.

[16] J. Denavit and R. S. Hartenberg, "A kinematic notation for lower-pair mechanisms based on matrices," Transactions of the ASME, vol. 22, pp. 215-221, 1955.

[17] MTC2, "Calibration specification for articulated arm coordinate measuring machines (JJF 1408-2013)," General Administration of Quality Supervision, Inspection and Quarantine of the People's Republic of China, 2013. 


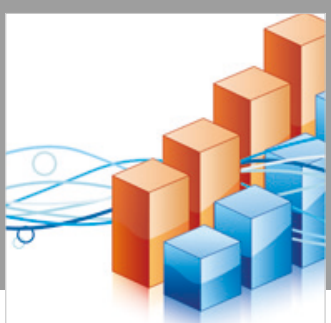

Advances in

Operations Research

\section{-n-m}
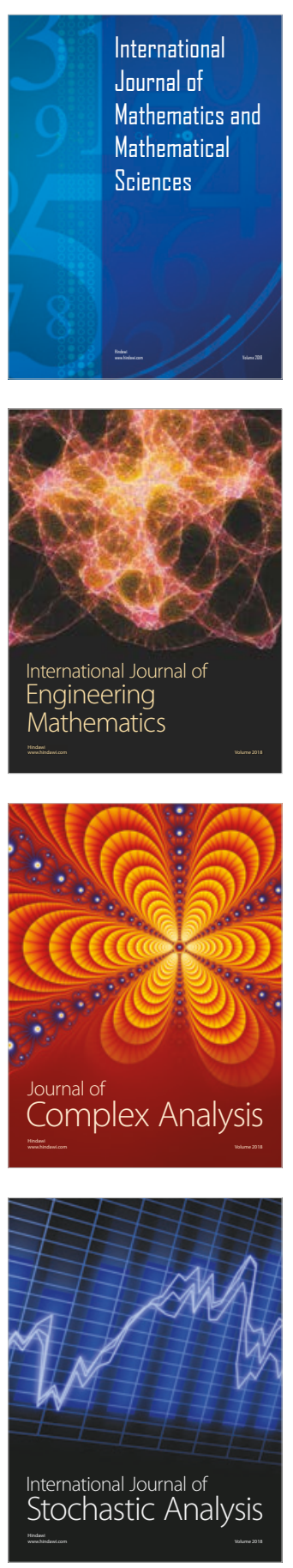
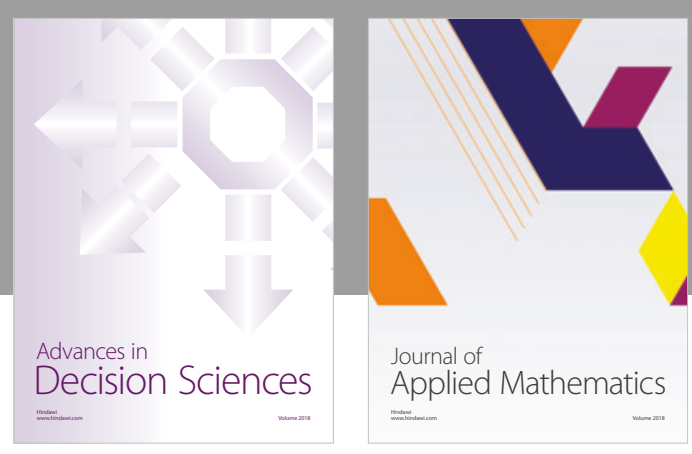

Journal of

Applied Mathematics
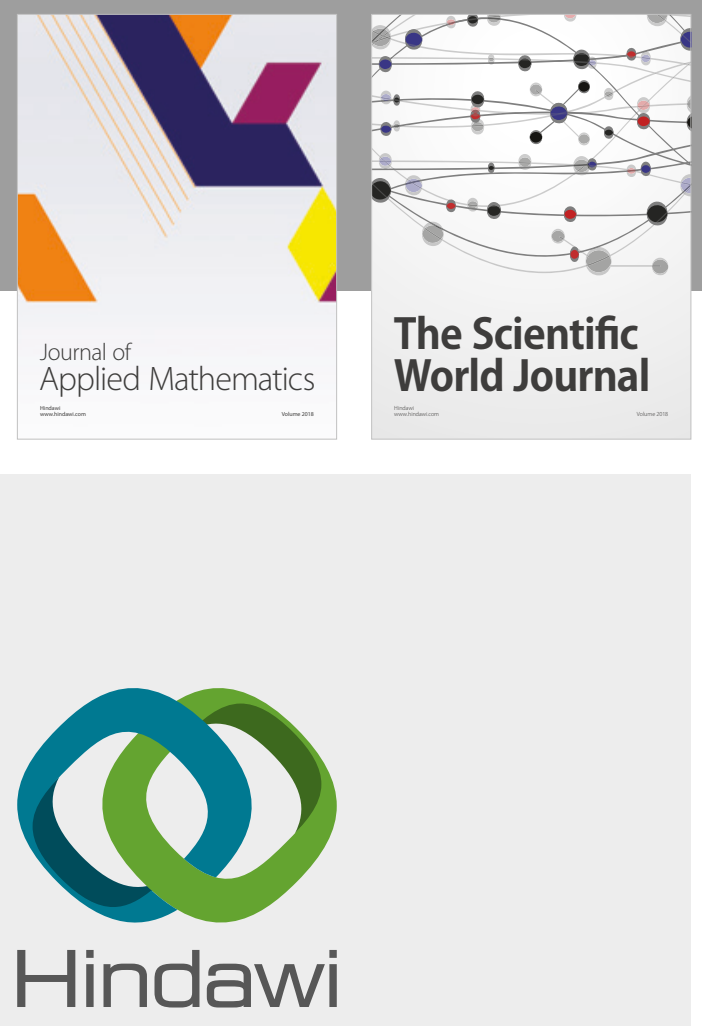

Submit your manuscripts at

www.hindawi.com

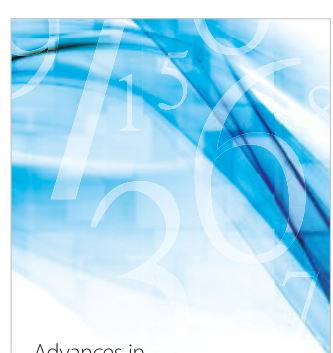

Advances in
Numerical Analysis
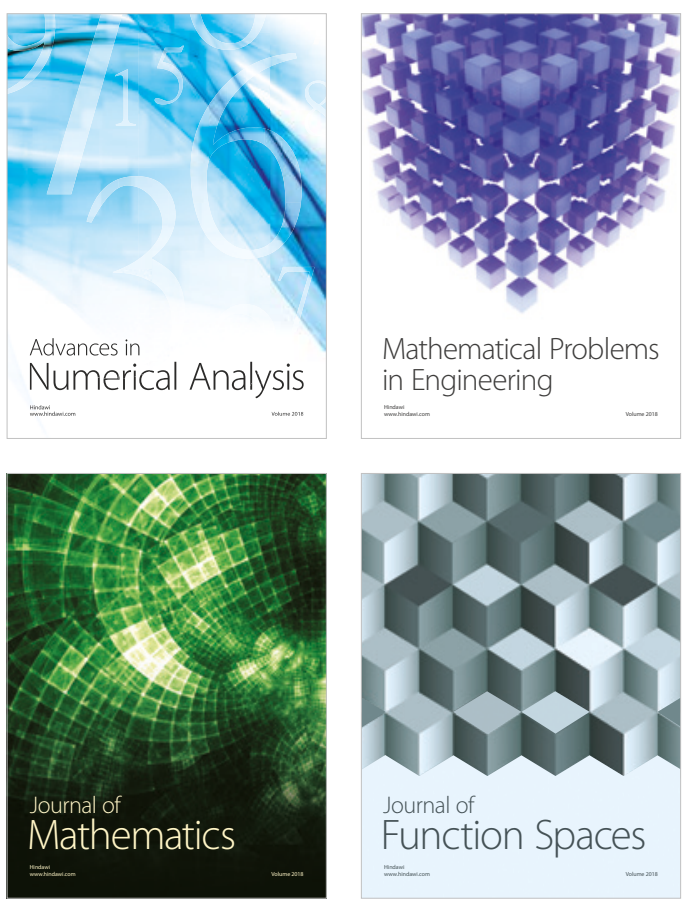

Mathematical Problems in Engineering

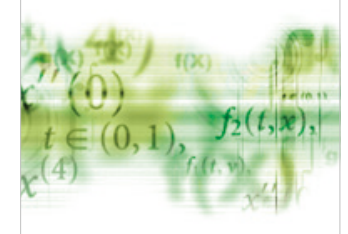

International Journal of

Differential Equations

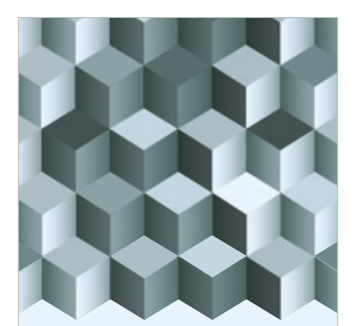

Journal of

Function Spaces

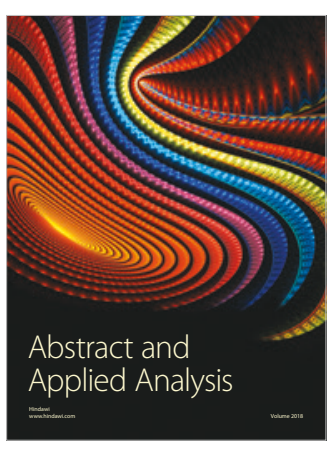

The Scientific

World Journal

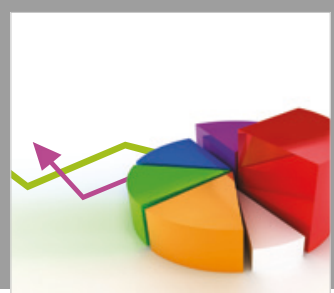

Journal of

Probability and Statistics
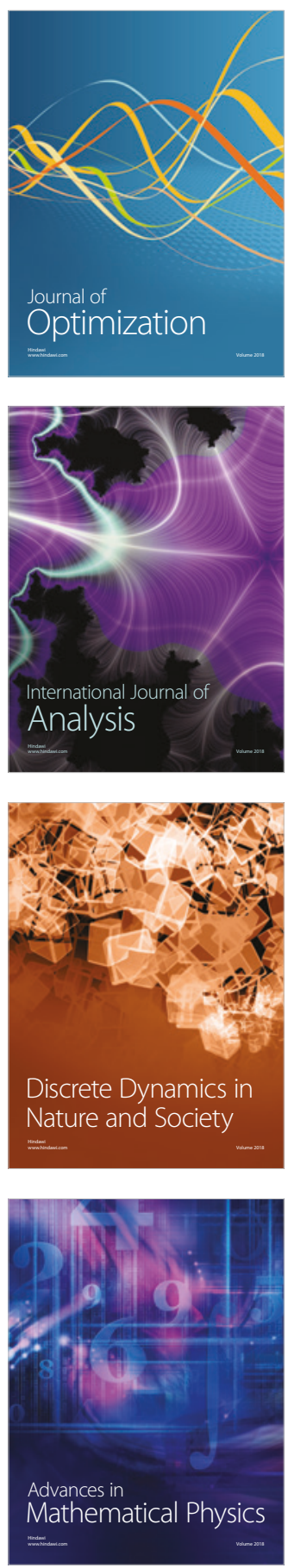